\title{
Angels in Focus ${ }^{1}$
}

\author{
David Atlas
}

Air Force Cambridge Research Laboratories, Sudbury, Mass.

(Received November 13, 1964)

\begin{abstract}
Recent independent observations of "dot" angel echoes are drawn together to provide a coherent picture of their physical structure. The angels act as point targets with durations proportional to beam width (for vertically pointing radars). The echoes are strongly coherent, indicating a smooth specular-like surface. Dual frequency measurements in the band 1 to $3 \mathrm{~cm}$ indicate that their radar cross sections are proportional to between the first and second power of wavelength. A range square dependence is also indicated. When tracked, the cross sections show strong enhancement at the zenith, decreasing roughly symmetrically on either side. Doppler measurements indicate that they almost always ascend with speeds of about $1 \mathrm{~m} \mathrm{sec}^{-1}$.

The observations are shown to be consistent with specular reflections from the hemispherical, concave-downward cap of a rising thermal or convective bubble. The specular point of the cap appears only briefly in a vertically pointing beam and so the echo appears to be from a point source. The concave-downward surface provides at least partial focusing, thus accounting for both the magnitude of the cross sections and their range-square dependence as the bubble expands with altitude. Furthermore, only rising bubbles have such favorably disposed upper surfaces. The dual frequency data indicate that the transition zone across the bubble cap is extremely sharp, of the order of $0.5 \mathrm{~cm}$. The sharp transition, the smoothness of the cap, and the slow ascent rates suggest that the flow is laminar.
\end{abstract}

\section{Introduction}

The "dot" or "point" angel is a transitory echo associated with an invisible atmospheric target passing through a fixed radar beam whose duration generally corresponds to the time of transport of a point source across the beam with the wind speed. Until recently, little else has been known about them and so their origin has defied adequate explanation. This paper gathers together a number of recent independent observations of "dot" angels from which we can now draw a coherent picture of their physical structure.

\section{Observations}

\subsection{Durations}

In addition to the older observations of Plank [1956], we now have data by Vrana [1961], Borchardt [1962], Hay and Reid [1962], and Ottersten (private communication) covering wavelengths from 0.86 to $10 \mathrm{~cm}$ and beam widths from 0.25 to $1.8 \mathrm{deg}$, all vertically directed. With one exception, the approximate maximum durations reported closely fit the expression $t=6 \theta$, where $\theta$ is the $3 \mathrm{~dB}$ beamwidth. Thus durations are directly proportional to beamwidth and correspond to point sources $(t=0$ when $\theta=0)$. Figure 1 shows simultaneous signal intensity records of an angel by Bor-

${ }^{1}$ This paper was presented at the World Conference on Radio Meteorology, Sept. 14-18, 1964, National Bureau of Standards, Boulder, Colo. chardt [private communication] using coaxial vertical radar beams at $0.86 \mathrm{~cm}(\theta=0.5 \mathrm{deg})$ and $3.2 \mathrm{~cm}$ $(\theta=1.8 \mathrm{deg})$. The ratio of the durations is about 3.3 , corresponding well to the ratio of the beamwidths. Usually the $0.86 \mathrm{~cm}$ angels are about $1 / 5$ as long as those at $3.2 \mathrm{~cm}$ because their echo intensities are smaller.

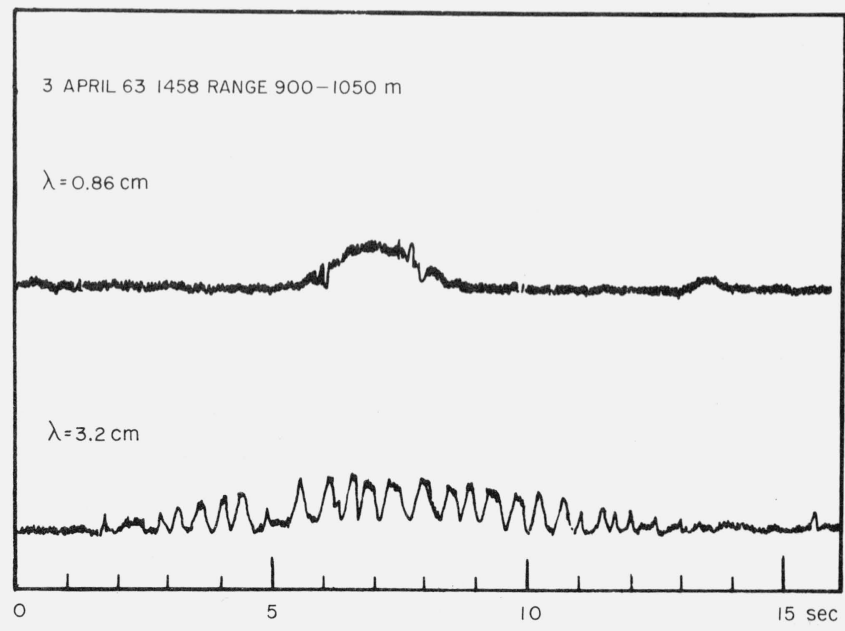

FigURE 1. Simultaneous recordings of point angel signal intensity versus time as the angels pass through the concentric vertically pointing beams of a $3.2 \mathrm{~cm}$ and a $0.86 \mathrm{~cm}$ radar with beam widths of 1.8 and 0.5 deg, respectively.

(Courtesy of H. Borchardt, Institute fur Mikrowellen, Mulheim/Ruhr, Germany). 


\subsection{Coherence}

The observations of Borchardt [private communication] (fig. 1) and of Vrana [1961] confirm the longknown fact that dot angels are strongly coherent with intensity-time patterns which are reasonable images of the radiation pattern. The coherence and the intensity pattern strongly indicate a smooth specularlike reflecting surface. Roelofs [1963] has tracked dot angels for periods up to $50 \mathrm{~min}$ and reports exceedingly coherent signals at high elevation angles, but less so the lower the elevation angle. Occasionally the $3 \mathrm{~cm}$ data of Borchardt [private communication] and Ottersten [1964] show a well defined beat frequency superimposed on the intensity trace as in figure 1 . The origin of this kind of modulation is not yet understood. It could arise in one of several ways: (a) two reflectors in relative radial motion; (b) two reflectors with constant spacing, but moving across the radar beam in such a manner as to find themselves alternately in and out of phase with one another; (c) one reflector moving radially but beating with the echo from a fixed target seen on a side lobe.

\subsection{Radar Cross Sections}

Borchardt [1962] took pains to adjust his coaxial radars to produce equal signals for targets of equal cross section; yet $3.2 \mathrm{~cm}$ angels ranged from 5 to 10 $\mathrm{dB}$ stronger than those at $0.86 \mathrm{~cm}$, indicating a $\lambda^{1}$ to $\lambda^{2}$ dependence. Vrana [1961] has plotted $0.86 \mathrm{~cm}$ angel cross sections versus altitude with the result shown in figure 2. While no one angel could be tracked up the axis of the beam, it seemed reasonable to assume that the strongest angels at each height would represent some kind of a "standard" angel target. Using an a priori assumption, I have put a $\sigma=k r^{2}$ curve through the strongest angel reported and find that this does, in fact, represent a reasonable upper bound for all the angels. Thus a range square dependence is suggested. Examination of the maximum cross sections reported by all observers shows that $\sigma_{\max } \approx 0.03 r^{2} \lambda^{2}(r-\mathrm{km}$, $\lambda-\mathrm{cm}, \sigma-\mathrm{cm}^{2}$ ) is a reasonable though rough approximation of the values attained.

Roelofs [1963] tracked dot angels with the $5.5 \mathrm{~cm}$ FPS-16 radar and noted the interesting dependence of average radar cross section upon elevation angle shown in figure 3 . Note that these are average profiles for all the angels on any one day. Also, none of them went directly overhead so that the curves are dashed near $90 \mathrm{deg}$ elevation. Obviously the angels are in optimum view when near the zenith and show a fair degree of symmetry on either side. The angular pattern is suggestive of a downward focusing effect.

\subsection{Motions}

Roelofs [1963] was usually able to track an angel for about $10 \mathrm{~min}$. Their speeds were in reasonable (though not perfect) accord with the winds. He also found that angels rise and fall with average speeds within $\pm 1 \mathrm{~m} \mathrm{sec}^{-1}$. In contrast, Battan [1963] found that dot angels, in his vertical Doppler radar beam, almost always rise with speeds of about $1 \mathrm{~m} \mathrm{sec}^{-1}$ or less.

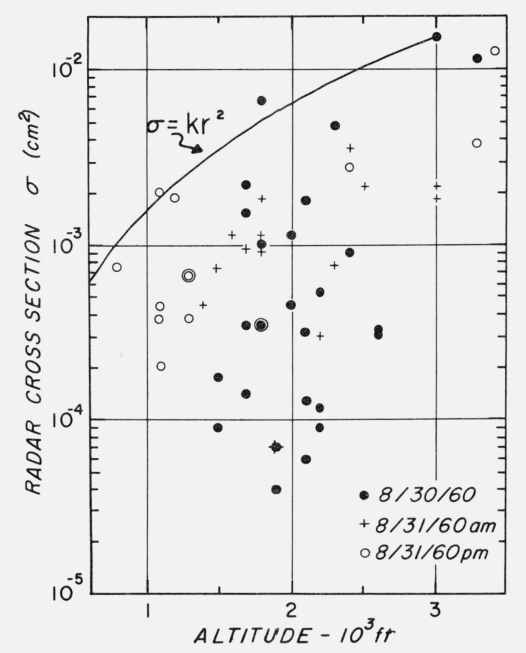

FIGURE 2. Cross sections of point angels recorded on the TPQ-6 vertically pointing $0.86 \mathrm{~cm}$ radar on Blue Hill, Boston, Mass., plotted versus range.

Curve indicates asymptote of maximum values and follows a range square law [after Vrana, 1961].

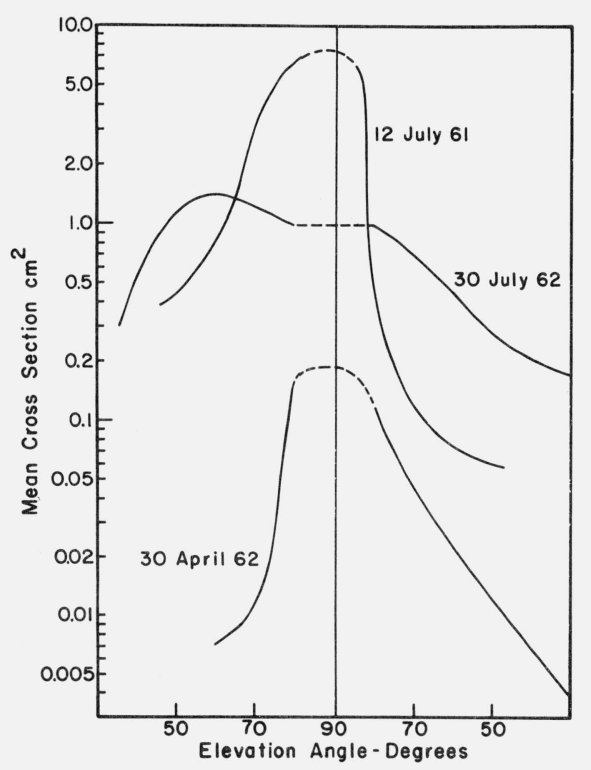

FIGURE 3. Average point angel cross sections as a function of elevation angle as recorded during tracking with the $5.5 \mathrm{~cm}$ FPS-16 on the days indicated.

90 degree is overhead. Angles left of center are for approaching angels; those to the right are for receding ones [after Roelofs, 1963]. 


\section{Model of the Dot Angel}

We shall see that the observations are in quite good accord with the model of a convective thermal or bubble shown in figure 4. Except for its smoothness, this model is virtually identical to the ring vortex bubble visualized by Scorer [1958] and others. The leading edge of the bubble is hemispherical in shape with a boundary which is smooth and sharply defined near the top and less well defined down along the sides. The vortex motion of the air within the bubble establishes and maintains the sharp gradient of refractive index at the cap [Malkus and Witt, 1959]. The bubble radius (a) expands with altitude $(R)$ along a well defined cone $a=k R$, where $k$ ranges from about $1 / 5$ to $1 / 2$ [Scorer, 1958]. Depending upon the stability of the environment, the bubble may flatten somewhat so that the radius of curvature near the cap may correspond to the condition $a=R$. The bubbles spewing from the top of a cumulus cloud have a virtual origin $(R=0)$ some place between cloud base and the ground, while the low level clear air thermals observed by Warner and Telford [1963] appear to have a virtual origin near or below ground level.

This model is consistent with the dot angel observations in the following ways: (1) Vertically pointing radars can receive specular reflections from the cap only when it is centered in the beam and so the angels appear as point targets; (2) Decreasing sharpness of the boundary refractive index gradient along the sides will decrease the signal intensities more or less symmetrically on both sides of the zenith as shown in figure 3 (increased roughness of the side boundaries would also account for the less coherent signals observed by Roelofs [1963] at low elevation angles);

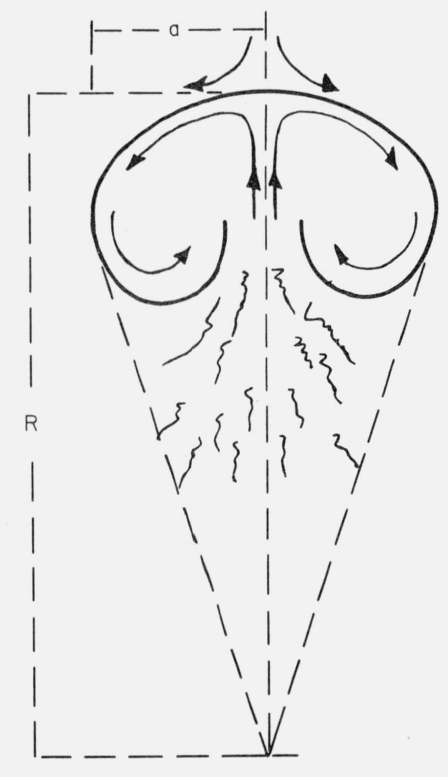

FiguRE 4. Model of a rising bubble.

Radius of curvature of cap denoted by $a$; height of cap above virtual origin is $R$.
(3) The concave downward cap provides at least partial focusing, permitting signal enhancement and accounting for the range square dependence of cross sections as the bubble expands (sec. 4); (4) A concave downward cap occurs only on a rising bubble, thus explaining why Battan's [1963] dot angels rarely fall. However, Roelofs [1963] could readily obtain a false downward velocity on a slowly rising but receding angel as his tracking radar locked on points successively farther down the side from the cap. Similarly, false horizontal velocities could be measured thus suggesting why angels don't always move with the observed winds.

\section{Theory}

\subsection{Cross Section of a Smooth Curved Surface}

We now inquire whether this model can account for the radar cross sections. The cross section of a smooth partially reflecting surface having two principal radii of curvature $a_{1}$ and $a_{2}$ at distance $r$ from the radar can be shown (F.S. Holt, private communication) to be

$$
\sigma=\pi a_{1} a_{2} \Gamma^{2} /\left[\left(1-a_{1} / r\right)\left(1-a_{2} / r\right)\right]
$$

where $\Gamma^{2}$ is the surface power reflection coefficient. The convention is that $a>0$ when the surface is concave toward the radar. For surfaces of revolution in which $a_{1}=a_{2}$,

$$
\sigma=\pi r^{2} \Gamma^{2} /[(r / a)-1]^{2}=\pi r^{2} G_{p} \Gamma^{2} .
$$

Since $\pi r^{2}$ is the specular cross section of a large metallic plane $(a=\infty), G_{p}=[(r / a)-1]^{-2}$ is the gain of the surface relative to that of a plane. The function $10 \log G_{p}$ is plotted in figure 5 , where we see that any concave surface with $a \geqslant r / 2$ has a radar cross section

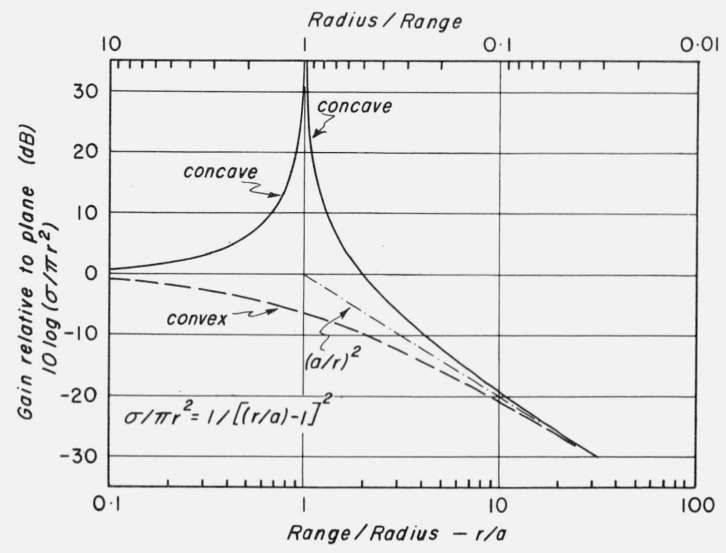

FIGURE 5. The cross section of a perfectly reflecting hemispherical shell of radius of curvature, a, (positive concave toward radar) at range, $\mathrm{r}$, normalized with respect to $\pi \mathrm{r}^{2}$, the cross section of a conducting plane.

Concave toward radar-solid curve; convex - dashed curve. 
greater than that of the plane and, when $a=r$, the cross section is infinite. This, of course, is the condition of perfect focusing. In the range $0.7 \leqslant r / a \leqslant 1.3$, $G_{p} \geqslant 10$ so that one need not be perfectly focused to obtain strong enhancement. Also, it will be seen from (1) that only one of the principal radii of curvature need be near the focusing condition to provide large cross sections.

For any rising bubble, we have seen that $a=k r$ ( $k=$ constant), so that $G_{p}$ is independent of range and $\sigma$ goes as the range square, thus confirming what we deduced earlier from Vrana's [1961] data (fig. 2).

\subsection{Power Reflection Coefficient}

In order to compute the absolute radar cross section, we must now estimate the power reflection coefficient, $\Gamma^{2}$. For a discontinuity which is very sharp with respect to the wavelength, $\Gamma^{2}=(\Delta N)^{2} \times 10^{-12} / 4$, where $N$ is the modified refractive index $(m-1) 10^{6}$. For a monotonic transition of $N$ such as that shown in the inset to figure 6 , the coefficient $\Gamma^{2}$ has been computed by Friend [1949] and is plotted there in decibels below the limiting value. The characteristic transition thickness $L$ represents the distance over which refractivity changes from 0.1 to 0.9 of the total change $\Delta N_{m}$. Note how sharply $\Gamma^{2}$ drops once the transition layer thickness $L$ exceeds the wavelength. The shape of the curve is altered somewhat for different forms of the $N$ transition, but its general features remain unchanged as long as the variation is monotonic. If we assume a value for the transition thickness (e.g., $L=0.5 \mathrm{~cm}$ ), then the abscissa becomes an inverse wavelength scale such as that plotted at the top and the curve represents the wavelength dependence of the cross section. Recalling that Borchardt's [1962] $3.2 \mathrm{~cm}$ angels exceeded the $0.86 \mathrm{~cm}$ cross sections by 5 to $10 \mathrm{~dB}$, we readily find that $L$ must fall between 0.3 and $0.43 \mathrm{~cm}$, and that $\Gamma^{2}$ is within $10 \mathrm{~dB}$ of its maximum value at $0.86 \mathrm{~cm}$, and within a 1 or $2 \mathrm{~dB}$ at $3.2 \mathrm{~cm}$.

\subsection{Values of the Cross Section}

Finally, if the bubble cap has a $=\mathrm{r} / 2$ (i.e., $G_{p}=1$ ), $L=0.43 \mathrm{~cm}$, and $\Delta N=20 N$ units $\left(\Gamma^{2}=10^{-10}\right.$ at 3.2 cm), then $\sigma=\pi r^{2} \times 10^{-10}$; i.e., $\sigma=3 \mathrm{~cm}^{2}$ at a range of $1 \mathrm{~km}$, easily accounting for the maximum angel cross sections at $3.2 \mathrm{~cm}$ wavelength. Roughness of the surface in the vicinity of the specular point would reduce the cross section, and we would have to call upon improved focusing to account for the observed values. This problem is treated more fully elsewhere [Atlas, 1964]. It is to be emphasized that essentially the same results would pertain if the reflecting surface included only a few Fresnel zones. Since we are usually concerned with short ranges, the main contributing region may be only a few meters in diameter. Thus, the entire thermal need not be so well shaped.

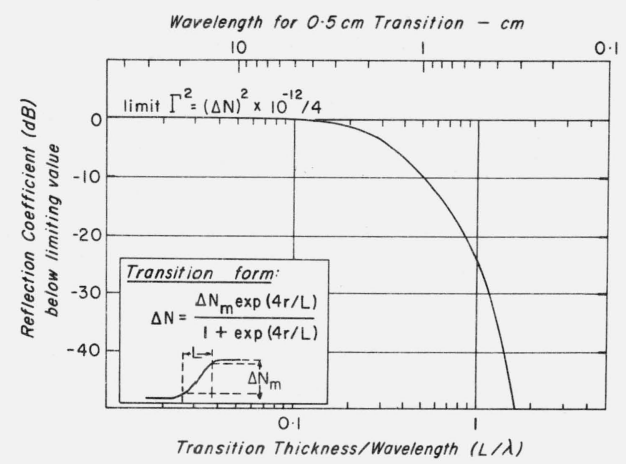

FIGURE 6. The power reflection coefficient of a partially reflecting plane surface at normal incidence having a refractive index profile indicated in the inset diagram, total index change $\Delta \mathrm{N}_{\mathrm{m}}$, and depth $\mathrm{L}$ (between 0.1 and $0.9 \Delta \mathrm{N}_{\mathrm{m}}$ ) normalized with respect to the sharp-edge coefficient $\left(\Delta \mathrm{N}^{2} / 4\right) \times 10^{-12}[$ after Friend, 1949].

\section{Discussion}

In general the physical model which we have used is well accepted among meteorologists. However, two features are implied by the theoretical treatment which are not generally acknowledged. These are (1) that the transition zone thickness across the cap be of the order of a wavelength or less and (2) that at least the first Fresnel zone (a few meters in diameter) centered on the specular point be smooth to a fraction of the wavelength.

Until recently we had no direct evidence of the possible existence of such sharp transitions. However, Lane [1964] has recently measured refractive index differences between two vertically spaced cavities of 3 to $4 N$ units in $12 \mathrm{~cm}$, and Turner and Hay [1963] have recorded changes of $7 N$ units in a vertical distance of $23 \mathrm{~cm}$. Smaller changes have been observed by the latter investigators to occur in a few centimeters. Thus, while we still lack the instruments to measure transitions of the order of a centimeter or less, we continue to find ever smaller transition zones as the resolving power of our instruments increases. Aircraft refractometer probes conducted by Brocks et al. [1963], simultaneous with the radar observation of angels have also shown the existence of sharpedged thermals or bubbles several hundred meters across with differences of 10 to $20 \mathrm{~N}$ units from the environment. Unfortunately the spatial resolution was of the order of 1 meter. Nevertheless, the occurrence of bubbles such as those visualized here in the same atmosphere in which angels occur is strong support for the thesis put forth here.

With regard to the required smoothness of the thermal cap, there is no direct supporting evidence. We can only reemphasize that such smoothness is implied by both the observed wavelength dependence and the strong coherence of the echoes. The implication is that the Reynolds number of the rising bubble is less than about $10^{5}$ and the flow around it is laminar 
rather than turbulent. In other words, the convection is gentle in accord with the slow ascent rates (sec. 2.4). For these reasons, the rough protuberances commonly seen on the boundaries of a cumulus cloud may have no counterpart on the gently rising bubble in clear air. Indeed, the need for a smooth wellshaped surface is also implied by the maximum occurrence of dot angel echoes on calm days and their disappearence when the surface winds exceed 20 to 25 knots [Plank, 1956; Hay and Reid, 1962].

A final word should be said about the possibility that the angels may be due to insects. Although the occurrence of insects in the diurnal and annual meteorological eycles is not unlike that of angels or of thermals, it would be virtually impossible to account for the wavelength dependence of $\lambda^{1}$ to $\lambda^{2}$ with insects smaller than those corresponding to the first resonant peak in the scattering curve for water spheres (i.e., $\bar{D} \leqslant 0.35 \lambda$ ). Thus, centimeter size insects would be required. Indeed, if insects scatter like water spheres, it would take a unique size to account for the wavelength dependence. On the other hand, if such large insects were responsible, the cross sections reported by both Vrana [1961] (see fig. 2) and Borchardt [1962] at 0.86 $\mathrm{cm}$ wavelength should be much larger. Finally, the cross sections of insects should show no range dependence whatsoever (fig. 2) and no preference for rising velocities [Battan, 1963]. Surely, insects will be detected by sensitive radars, but they will not behave in the manner indicated.

(Paper 69D-519)

\section{References}

Atlas, D. (1964), Advances in radar meteorology, Advances in Geophysics, 10 (Academic Press, New York, N.Y.) in press.

Battan, L. J. (1963), The vertical velocities of angel echoes, Proc. Tenth Weather Radar Conf., Boston, Amer. Meteor. Soc. 309-315.

Borchardt, H. (1962), Wolkenbeobachtungen mit einem doppelwelligen Radargerat, Beitrage zur Physik der Atmosphare 35, No. 1/2, 43-68.

Brocks, K., G. Fengler, and H. Jeske (1963), Models of the troposphere from direct measurements of the atmospheric refractive index, Radiometeorological Papers, 7, Institute for Radiometeorology and Maritime Meteorology, University of Hamburg, 25-49.

Friend, A. W. (1949), Theory and practice of tropospheric sounding by radar, Proc. IRE 37, 116-138.

Hay, D. R., and W. M. Reid (1962), Radar angels in the lower troposphere, Can. J. Phys. 40, 128-138.

Lane, J. A. (1964), Some measurements of the structure of elevated layers in the troposphere, Proc. 1964 World Conf. on Radio Meteorology, Boulder, Colorado, Amer. Meteor. Soc. Boston, 248-251.

Malkus, J. S., and G. Witt (1959), The evolution of a convective element: A numerical calculation. The atmosphere and the sea in motion, 425-439 (The Rockefeller Institute Press, New York, N.Y.).

Plank, V. G. (1956), A meteorological study of radar angels, Geophys. Res. Paper No. 52, Air Force Cambridge Research Labs. p. 117.

Roelofs, T. H. (1963), Characteristics of trackable radar angels, Center for Radiophysics and Space Research, Report No. 137, Cornell Univ. p. 51.

Scorer, R. S. (1958), Natural aerodynamics, p. 313 (Pergamon Press, New York, N.Y.).

Turner, H. E., and D. R. Hay (1963), Fine structure of temperature and refractivity in the lower troposphere, Can. J. Phys. 41, 1732-1737.

Vrana, N. (1961), Some characteristics of radar angel echoes, Center for Radiophysics and Space, Research Report No. 32, Cornell Univ. p. 29.

Warner, J., and J. W. Telford (1963), Some patterns of convection in the lower atmosphere, J. Atmospheric Sci. 20, No. 4, 313-318. 